\title{
Drag reduction and shear resistance properties of ionomer and hydrogen bond systems based on lauryl methacrylate
}

\author{
Yu Ping, Li Changyu, Zhang Changqiao*, Chen Shiwei, Fang Shu and \\ Sun Hui
}

School of Chemistry and Chemical Engineering, Shandong University, Jinan 250061, China

(C) China University of Petroleum (Beijing) and Springer-Verlag Berlin Heidelberg 2011

\begin{abstract}
Based on molecular dynamics simulation results, a lauryl methacrylate polymer with drag reduction and shear resistance properties was designed, and synthesized by emulsion polymerization using 2-vinyl pyridine and methyl methacrylate as the polar polymerization monomer. After ionization of lauryl methacrylate polymer, an ion-dipole interaction based drag reduction agent (DRA) was obtained. The existence of ion-dipole interaction was proven through characterization of the drag-reducing agent from its infrared (IR) spectrum. The pilot-scale reaction yield of the DRA under optimum conditions was investigated, and the drag reduction and shear resistance properties were measured. The results show that: 1) The ion-dipole or hydrogen bonding interaction can form ladder-shaped chains, therefore the synthesized DRA has shear resistance properties; 2) The larger the molecular weight (MW) and more concentrated the distribution of MW, the better the drag reduction efficiency and the performance of the ionomer system was superior to that of the hydrogen bonding system; 3) With increasing shear frequency, the drag-reduction rates of both the DRAs decreased, and the drag reduction rate of the ionomer system decreased more slowly than of the corresponding hydrogen bonding system. From the point of view of drag reduction rate and shear resistance property, the ionomer system is more promising than the hydrogen bonding system
\end{abstract}

Key words: Molecular design, ion-dipole interaction, hydrogen bonding, drag reduction rate, shear resistance property

\section{Introduction}

Pipelines are one of the five basic transportation modes (waterway, railway, highway, pipeline and air transportation). The total mileage of pipeline transportation has exceeded that of railways worldwide. In the oil industry, about 93 percent of crude oil is transported by pipeline (Sun,1996). However, pipeline transportation has the problems of long transportation distance, large diameter of pipelines and high energy consumption. Particularly, due to the aging and corrosion of long-term service pipelines, operation under original design pressure or increased pressure for normal transportation may cause serious safety problems. To overcome these problems, use of drag reduction technology is a good countermeasure, i.e., adding a small amount of specific compounds in the system to reduce the resistance of fluids (Motier and Prilutski, 1985). These specific compounds are called drag reduction agents (DRA).

Under the designed temperature condition, long distance

*Corresponding author. email: zhangchqiao@sdu.edu.cn

Received November 11, 2010 pipeline transportation should be pressurized by pumps to maintain the pressure difference of the whole pipeline in a reasonable range. These pumps are usually called pipeline transportation stations, which is a standard continuous transmission mode. Oil-soluble drag reducing agents are widely used in oil transportation. They can be used to maintain the designed capacity at decreased pressure, or used to enhance the transport capacity (Liu et al, 2008). As one important type of DRA, poly- $\alpha$-alkene has been used in actual pipeline transportation for many years. However, due to its comb-like structure, the backbone chains of $\alpha$-olefins can be easily broken when they pass through the pumps in the pipeline transportation stations, and the drag reduction efficiency is greatly decreased. Therefore, developing a new type of DRA with high drag reduction efficiency and shear stability has become an important topic in study of oilfield chemicals (Mei, 2005).

Based on the previous studies (Pang and Zhang, 2009), a new type of DRA is designed and synthesized in our work in laboratory scale and scale-up (Shui et al, 2008; SY/T65782009), in which ion-dipole and hydrogen bond interactions exist. These DRAs are expected to have high drag reduction 
efficiency and shear resistance. In addition, the effect of Reynolds number (Resende and Escudier, 2006) and molecular weight (Zhao et al, 2007) of the polymer on drag reduction rate were also investigated.

\section{Molecular design and experimental}

Polymer drag reducer is synthesized through copolymerization of different carbon numbers of alpha olefin. Its main and branched chains are all combined by covalent bonds, and the molecular weight is mostly more than 4 million. When the polymer drag reducer flows through a high-shear area, covalent bonds are broken under the high shear force and the drag reducer loses its efficiency. The aim of designing a shear-resistant drag reducer molecule is to synthesize a cluster system or hydrogen bond system. Because of the ion-dipole effect of a cluster system and the hydrogen bonding effect of hydrogen bond system, these both systems not only have excellent drag reduction performance but also display shear resistance properties. When the polymer drag reducer flows through a high-shear area, because the covalent bonds are stronger than those of ion-dipole and hydrogen bond, the latter are broken more easierly than covalent bonds, reducing the effect of shear strength damage on the chain structure. When the polymer drag reducer flows into a low-shear area, the ion-dipole or hydrogen bond is reassociated automatically, and its drag reduction properties are recovered. Thus the drag reducer has the dual function of shear resistance and drag reduction.

The idea for designing a shear-resistant drag reducer molecule is that when good drag reduction performance is required, an ion-dipole cluster system or a hydrogen bond system should be used. Therefore, we first conducted molecular dynamics simulation to study the possible microscopic structure and dynamic properties of both systems. The molecular dynamic simulation was carried out in the isobaric-isothermal (NPT) system using the all-atom force field based on OPLS-AA/AMBER force field (Liu et al, 2010). The pressure was 1 bar with a compressibility of $4.5 \times 10^{-5} \mathrm{bar}^{-1}$. A leap-frog algorithm was used to integrate Newton's equations of motion, with a time-step of 1 fs and the long-range electrostatic interaction was calculated by using the particle mesh Ewald method with a cut-off radius of 1.2 $\mathrm{nm}$. The model system for the MD simulations is composed of 252 cations and 252 anions, total containing 7308 atoms. These cations and anions were all arbitrarily distributed in a rectangular box with a low density and periodic boundary conditions. Using the steepest descent method for minimizing energy and the $500 \mathrm{ps}$ balance simulation, the box is compressed to normal size, then the normal sized structure was used in balance simulation. All the simulations were carried out using the GROMACS 3.3.3 software package.

Beginning with the dipole cross linking formed by the iondipole effect (Peiffer, 1987), the microstructure and molecular dynamics of monovalence pyridine were calculated. Based on this, we investigated the similar structure and behavior of hydrogen bonds. When the monovalence pyridine, which has a nitrogen $(\mathrm{N})$ atom, reacts with another compound to form a system, the electric behavior of nitrogen atom is related to the property of the compound. When it reacts with fluorine (F)-containing compounds whose chemical structure is very stable, the nitrogen atom can easily lose an electron to create a positive ion. When it reacts with other compounds, the nitrogen atom gains an electron and creates a negative ion. In order to simplify the calculation of molecular dynamics simulation, and conveniently obtain the calculation parameters from literature, n-Butyl pyridine (BPy) is selected as a typical representative of monovalence pyridine, and the other compound is $\mathrm{BF}_{4}$, making the system provide cationic $\mathrm{N}$ and $\mathrm{BF}_{4}^{-}$. Considering the environmental factors and industrial cost of fluoride (Zhu et al, 2007), if the monovalence pyridine is ester-olefins and the other compound is non-fluoride, then the nitrogen on the pyridine ring is negative and the nonfluoride is positive. For the system composed of ester-olefins and non-fluoride, the molecular dynamics simulation results are still suitable for the ester-olefins and non-fluoride system as that for the $\mathrm{BPy}-\mathrm{BF}_{4}$ system, except for the difference of the cation and anion.

Based on the molecular dynamics simulations, the cationcation, cation-anion, and anion-anion center-of-mass radial distribution functions (RDFs), (RDFs), $g(r)$, are calculated and the results are shown in Fig. 1. Clearly, we can see that all RDFs show strong and damped oscillations with and beyond $1.8 \mathrm{~nm}$, respectively, which indicate a long-range ordered structure. For further insight into the structural features of the cation and the anion, the spatial distribution of the anions and cations around a given cation are also shown in Fig. 2. Seen from this figure, the anions are mainly distributed near the $\mathrm{N}$ atom of the pyridine cation. The diffusion coefficients of n-butyl pyridine cations ([BPy $\left.]^{+}\right)$and boron tetrafluoride anions $\left(\left[\mathrm{BF}_{4}\right]^{-}\right)$are shown in Fig. $3 . \mathrm{MSD}\left(\mathrm{A}^{2}\right)$ is the diffusion coefficients, $\beta$ is $\log \left[\operatorname{MSD}\left(\mathrm{A}^{2}\right)\right]$ and $\gamma$ is gauss of $\operatorname{MSD}\left(\mathrm{A}^{2}\right)$. The anions and cations of the BPy-BF4 system form a strong dipole force, and their diffusion coefficients are similar to each other. These results indicate that the monovalence pyridine with $\mathrm{N}$ atoms monomer is the direction for molecular design of ionomer system drag reducer. For hydrogen system drag reducer, except for the non-ionized hydroxyl, its microstructure and dynamic properties are similar to the ionomer system.

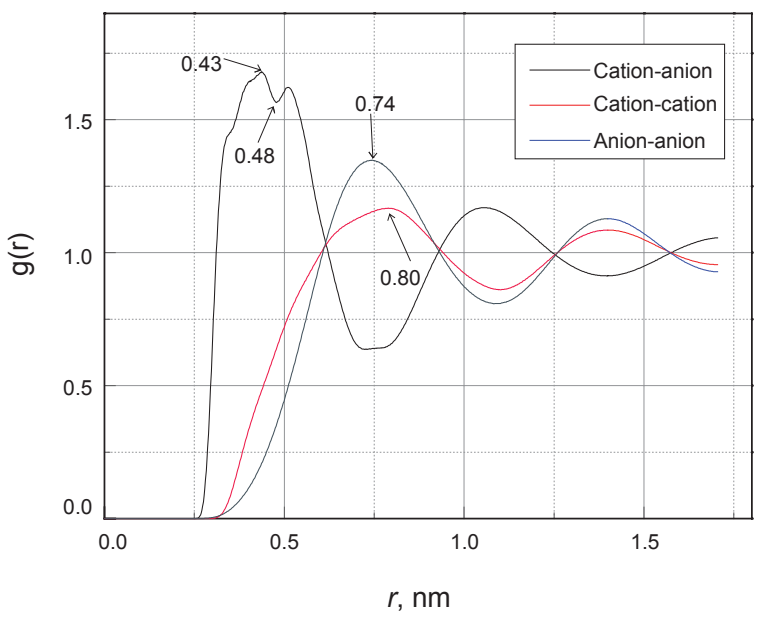

Fig. 1 Radial distribution function between $[\mathrm{BPy}]^{+}$and $[\mathrm{BF} 4]^{-}$ Note: $g(r)$ is anion-anion center-of-mass radial distribution functions 


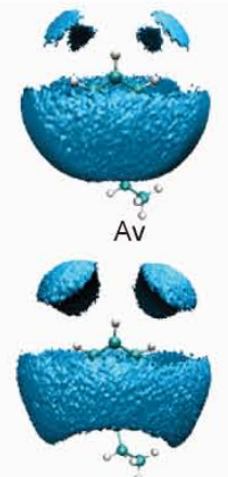

$\mathrm{Bv}$

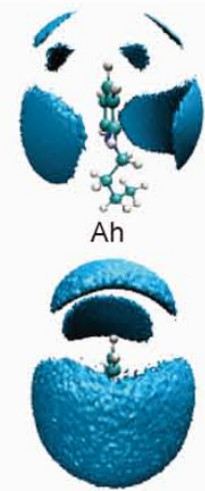

$\mathrm{Bh}$

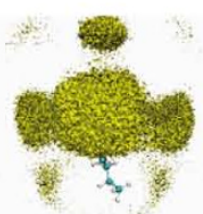

Cv

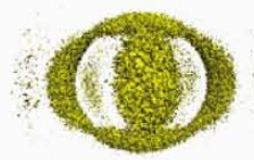

$\mathrm{Ch}$

II

Fig. 2 Spatial distribution for I and $\mathrm{II}$ : (A) anions around cations at 0-0.48 nm with blue isosurfaces, (B) anions around cations at $0.48-0.73 \mathrm{~nm}$ with blue isosurfaces and (C) cations around anions at $0.48-0.73 \mathrm{~nm}$ with yellow isosurfaces, where subscripts $\mathrm{v}$ and $\mathrm{h}$ stand for the vertical and horizontal views of the pyridine ring plane, respectively

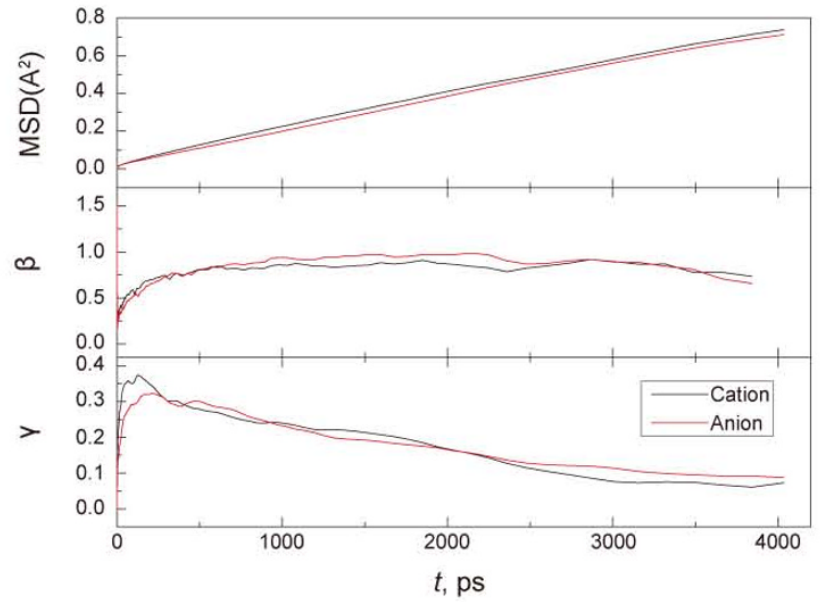

Fig. 3 The diffusion coefficients of $[\mathrm{BPy}]^{+}$and $[\mathrm{BF} 4]^{-}$ Notes: $\operatorname{MSD}\left(\mathrm{A}^{2}\right)$ is the diffusion coefficients, $\beta$ is $\log \left[\operatorname{MSD}\left(\mathrm{A}^{2}\right)\right]$ and $\gamma$ is gauss of $\operatorname{MSD}\left(\mathrm{A}^{2}\right)$

Thus there are two types of compounds selected for an ionomer system drag reducer: One is a long-chain compound with a monovalence pyridine functional group, and the other is a long-chain compound with more cations in cluster reaction. In the ionomer system, the monovalence pyridine functional group and hydroxyl groups can produce an iondipole effect, achieving a ladder-shaped link between the two long-chain compounds. The compounds for the hydrogen bonding system are similar to that for ionomer system except for the hydroxyl groups in their long-chain compound with anions are not ionized.

A terpolymer with a pyridine group was synthesized from lauryl methacrylate, styrene, and vinyl pyridine. A binary copolymer with hydroxyl group that can be ionized was synthesized from lauryl methacrylate and acrylic acid. These two copolymers were mixed uniformly to form the ionomer system DRA. Fig. 4 shows the ion-dipole interaction between the two copolymers.
When the two types of polymer compounds were mixed together, the monovalence pyridine group reacted with the ionized hydroxyl group to produce a strong ion-dipole effect to form a ladder-shaped chain. When the DRA flows through a high-shear zone (Mowla and Naderi, 2006), due to the existence of ladder-shaped chain formed by iondipole effect, the shear stress was distributed from one polymer chain to two polymer chains. Because the iondipole force is weaker than the covalent bonding force, the ion-dipole bond is easier to rupture than covalent bonds. These two reasons led to a decrease of shear stress on the polymer chain, so it reduced the number of fractured covalent bonds. When the drag reducer flowed into a lowshear area, the ion-dipole and hydrogen bond were reassociated automatically to generate two functions of drag reduction and shear resistance. For the hydrogen system, the hydrogen bonds had a similar function of ion-dipole interactions.

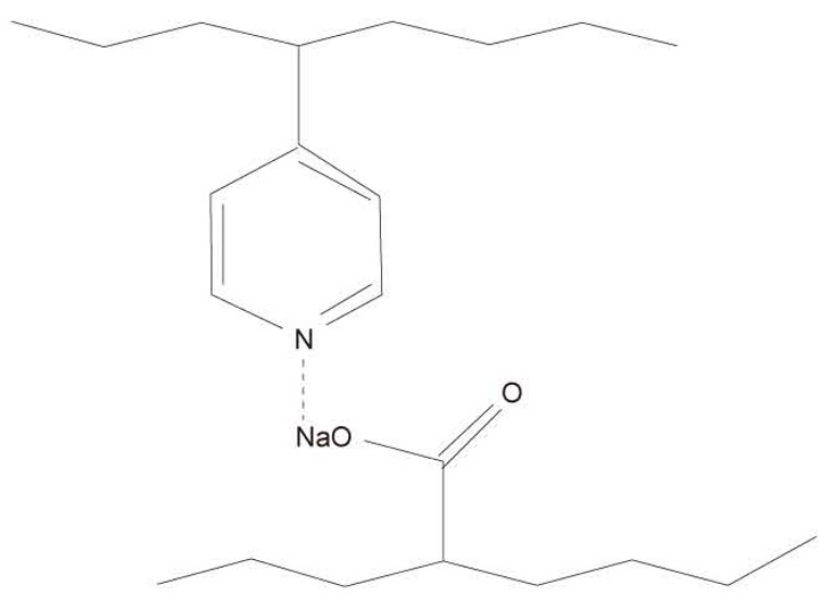

Fig. 4 The scheme of ion-dipole interaction 


\section{Synthesis and experimental apparatus}

\subsection{Chemicals and experimental apparatus}

Chemicals: Methyl methacrylate (Tianjin Hongyan Chemical Co. Ltd); Phenylethylene (Shanghai Chemical Co. Ltd ); Lauryl methacrylate, 2-vinyl pyridine (ACROS); Persulfate (Beijing Chemical Co. Ltd); Methanol (Jinan No. 2 Petrochemical Plant); Ethanol (Tianjin Fuyu Fine Chemical Co. Ltd); Toluene (Laiyang Kangde Chemicals Co, Ltd).

The terpolymer and the binary copolymer were synthesized by the emulsion polymerization method. Fig. 5 gives the experimental apparatus for emulsion polymerization.

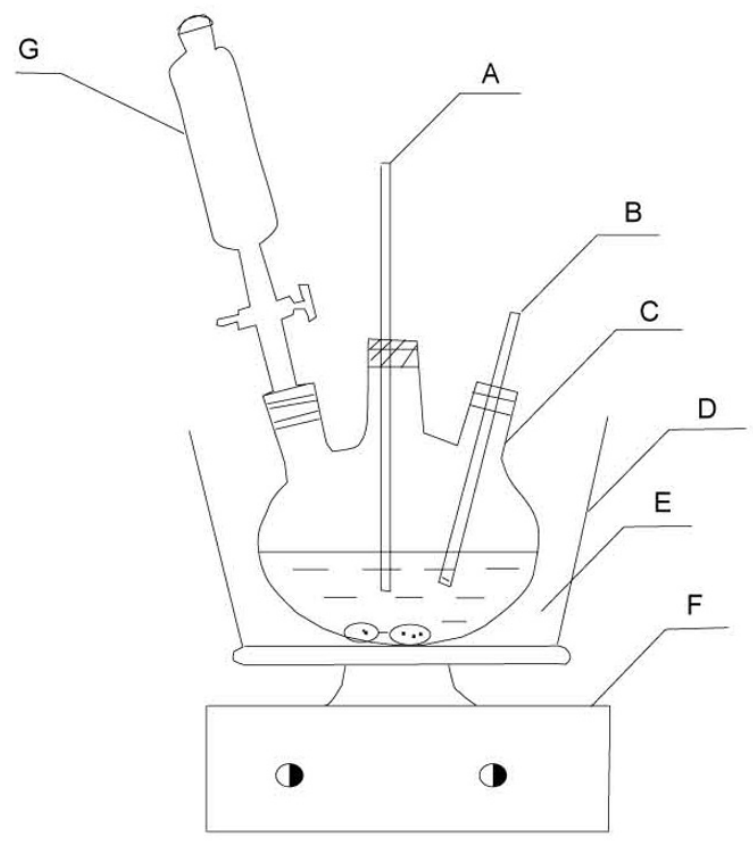

Fig. 5 Experiment apparatus for emulsion polymerization A. thermometer, B. nitrogen pipe, C. three neck flask, D. water-bath pot, E. water, F. constant temperature magnetic blender, G. dropping funnel

\subsection{Preparation of ionomer system and hydrogen bond system}

A binary copolymer of a proton donor polymer (bcPDP) was synthesized by the reaction of acrylic acid (2 $w t \%)$ and laury methacrylate (98 wt \%) through emulsion polymerization, with phenyl ether as the emulsifier and persulfate as the initiator under continuous stirring at $45^{\circ} \mathrm{C}$ for 12 hours. Then, ethanol was added to the emulsion. After precipitation, separation, and vacuum drying at $60{ }^{\circ} \mathrm{C}$, PDP was obtained.

The ternary copolymer of protons acceptor polymer (tcPAP) was synthesized by a similar route for bc-PDP except for that the reactants were lauryl methacrylate (58 wt\%), styrene (40 wt $\%$ ), and vinyl pyridine (2 wt \%) and the reaction temperature and time were $30^{\circ} \mathrm{C}$, and $14 \mathrm{~h}$, respectively.

The PDP was ionized by using methanol containing $\mathrm{NaOH}$. Then the ionized PDP was mixed with PAP (with a mass ratio of $1: 1)$ to form the ionomer system DRA. However, the hydrogen bond system DRA was obtained by mixing PAP and PDP (mass ratio of 1:1) without ionization of PDP.

The ionomer system DRA and PDP containing hydroxyl group were characterized by using VERTEX-70 infrared spectrometer (Shimadzu Corp., Japan). The infrared spectrum is shown in Fig. 6.

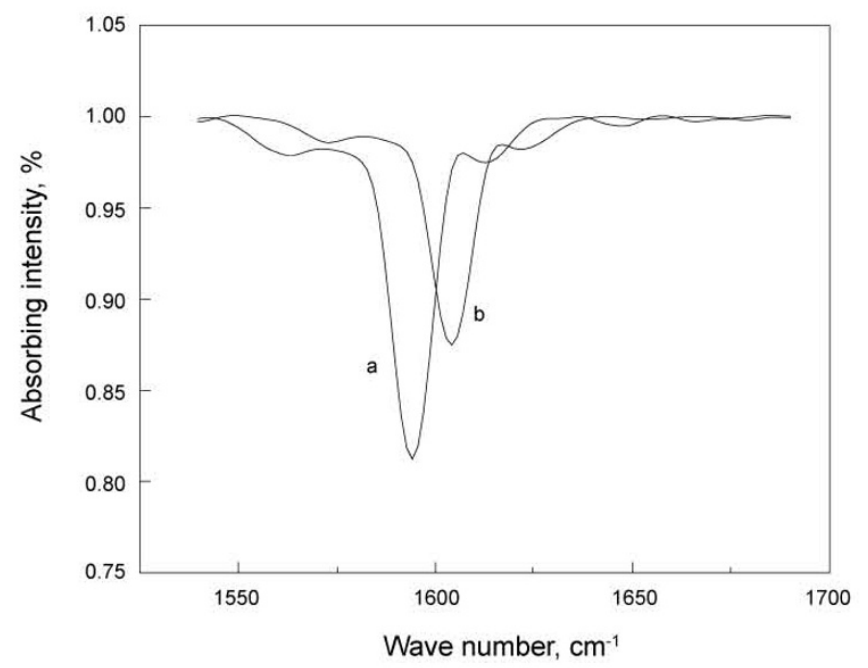

Fig. 6 IR spectra of PDP (a) and the mixture of PDP/PAP (b)

From Fig. 6, it can be seen that the two IR absorption peaks of C-N in PDP and ionomer systems are 1,594 and 1,605 $\mathrm{cm}^{-1}$, respectively. The carbon chain of the PDP containing sodium ions can form an ion-dipole with the nitrogen atom of 2-vinyl pyridine in the DRA. The IR absorption frequency of this ion-dipole interaction is higher than that of hydrogen bonding.

In addition, dynamic scattering characterization was carried out by using DAWN HELEOS 18-angle laser light scattering instrument (Wyatt Company, USA). The test condition was: $\lambda_{0}=658 \mathrm{~nm}$, scattering Angle range of $0-90^{\circ}$, with pure tetrahydrofuran as solvent (Deng and Liang, 2010). The hydrodynamic radius of bc-PDP, ionized tc-PAP and the ionomer system DRA were studied. The laser light scattering test results are shown in Fig. 7.

The results in Fig. 7 indicated that the hydrodynamic radius of bc-PDP is $67 \mathrm{~nm}$, and ionized tc-PAP is $50.7 \mathrm{~nm}$. However the ionomer system DRA is $112.3 \mathrm{~nm}$, which is obviously higher than bc-PDP and tc-PAP, indicating the existence of ion-dipole interaction between bc-PDP and ionized tc-PAP, through which molecular clusters are formed. 


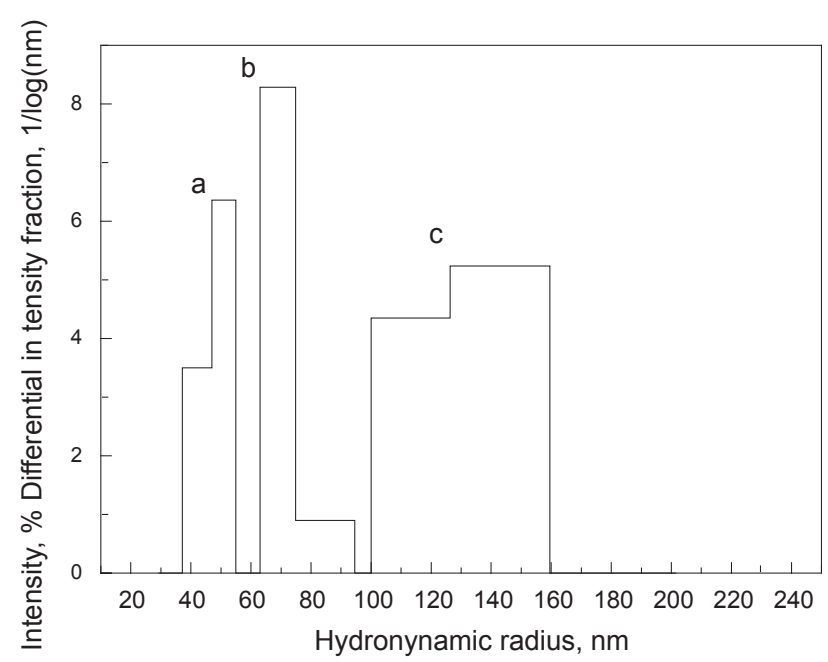

Fig. 7 The hydrodynamic radius of polymer (a) bc-PDP, (b) tc-PAP, and (c) ionomer system DRA

\section{Drag reduction properties of ionomer and hydrogen bond systems}

The DRA testing and evaluation system was used according to the "Lab test method for the performance of drag reduction agents to be applied in oil pipeline" (from China Petroleum Industry Standard SY/T6578-2009) and is shown in Fig. 8, with the $0^{\#}$ diesel oil as the solvent (Wilkens and Thomas, 2007). The drag reduction performance of the two systems were compared.

\subsection{Effect of Reynolds number of oil fluid on drag reduction performance}

Drag reduction performance testing was carried out in a $15 \mathrm{~mm}$ diameter circular pipeline at different Reynolds numbers and pressures such as $0.05,0.1,0.2,0.3 \mathrm{MPa}$. Table 1 shows the relationship between pressure and pipeline parameters and Fig. 9 shows the relationship between drag reduction and Reynolds number based on Table 1.

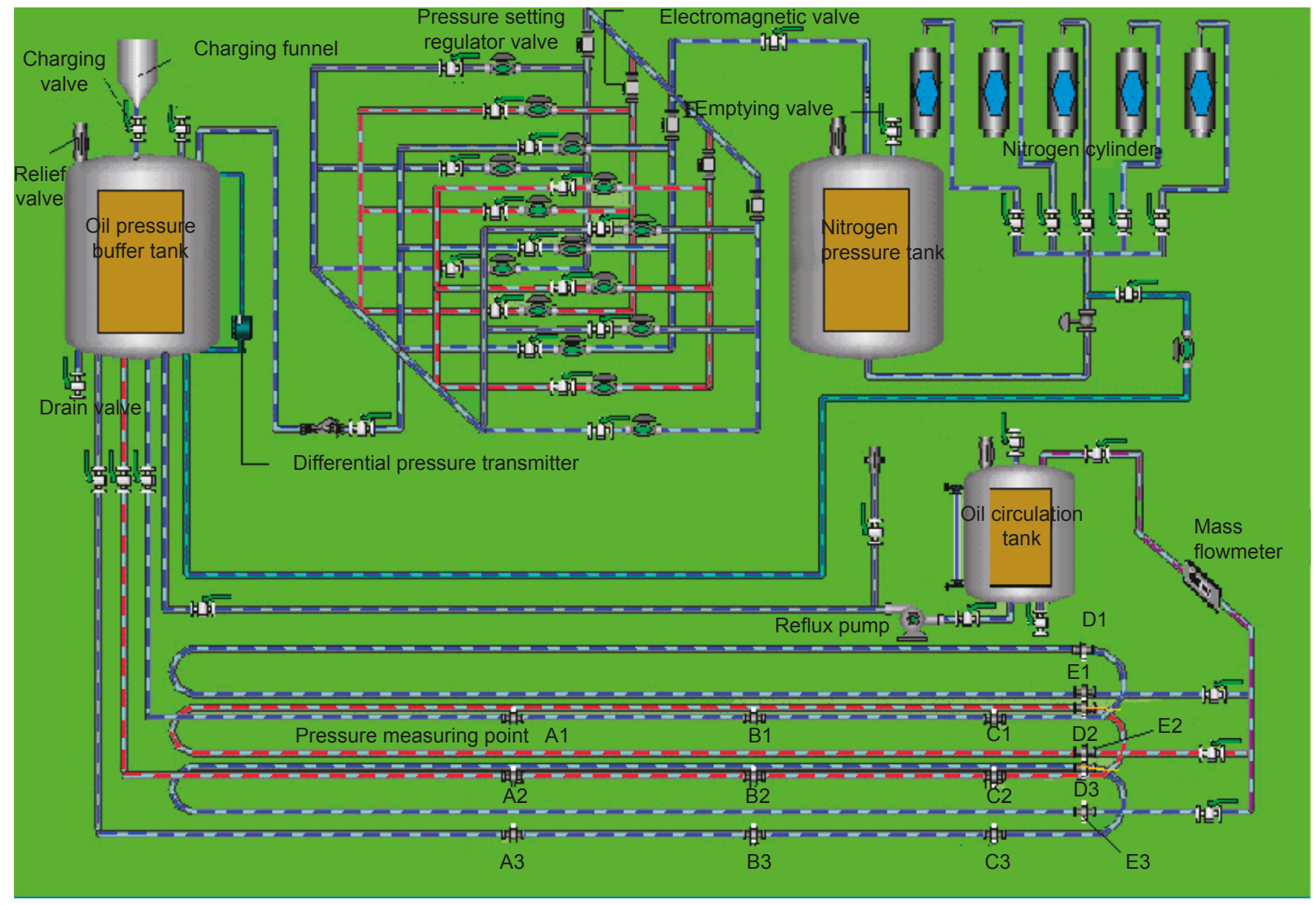

Fig. 8 The test unit for drag reducing agent

Table 1 The relationship between pressure and pipe parameters

\begin{tabular}{ccccc}
\hline \multirow{2}{*}{ Pipeline parameters } & \multicolumn{3}{c}{ Pressure } \\
\cline { 2 - 5 } & $0.05 \mathrm{MPa}$ & $0.10 \mathrm{MPa}$ & $0.20 \mathrm{MPa}$ & $0.30 \mathrm{MPa}$ \\
\hline Velocity, $\mathrm{m} / \mathrm{s}$ & 0.44 & 0.95 & 1.54 & 1.89 \\
Flow, L/min & 4.66 & 10.10 & 16.32 & 20.03 \\
Re number & 3581 & 7732 & 12534 & 15383 \\
\hline
\end{tabular}




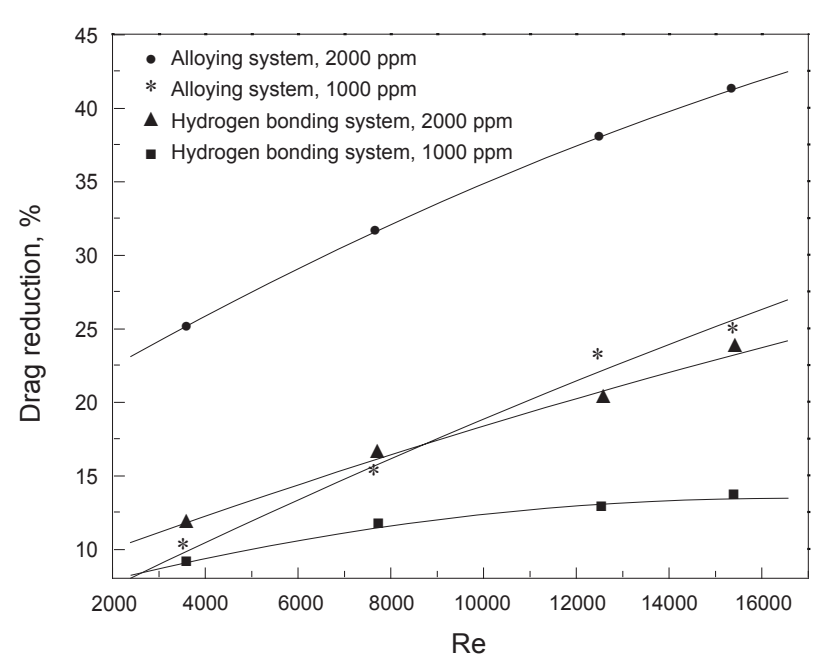

Fig. 9 The drag reduction vs. Reynolds number

As shown in Fig. 9, the drag reduction rate of the ionomer system and the hydrogen bonding system both increased with Reynolds number. For 1,000 ppm addition, the drag reduction performance of the both systems was similar at low Reynolds number, but the difference in their drag reduction performance became larger with increasing Reynolds number. For $2,000 \mathrm{ppm}$ addition, the drag reduction performance of the both systems showed a large difference at low Reynolds number, and the situation remained unchanged with increasing Reynolds number. The relationship between drag reduction performance and Reynolds number indicated that the ionomer system is superior to the hydrogen bonding system.

\subsection{Effect of polymer concentration on drag reduction performance}

Since the drag reduction performance is strongly influenced by the polymer concentration, the throughput increasing capacity (TI) of oil with increasing concentration at a Reynolds number of 15,383 and $25^{\circ} \mathrm{C}$ between the ionomer system and the hydrogen bond system was measured. Subsequently, the drag reduction rate was calculated by TI. The relationship between drag reduction and polymer concentration is shown in Fig. 10.

Fig. 10 shows that the drag reduction rate of the hydrogen bonding system increased with increasing polymer concentration, while for the ionomer system, the drag reduction rate changed very little over $2,000 \mathrm{ppm}$, although it increased with increasing polymer concentration when the polymer concentration smaller than $2,000 \mathrm{ppm}$. This is because the reduction rate increases with the drag reduction agent's concentration increasing. When the drag reduction system reaches its critical concentration, the reduction rate no longer increases. At the experimental concentrations, the drag reduction rate of the ionomer was improved more than that of the hydrogen bond system.

\subsection{Molecular weight of polymer on drag reduction performance}

For the functional polymers, the molecular weight and

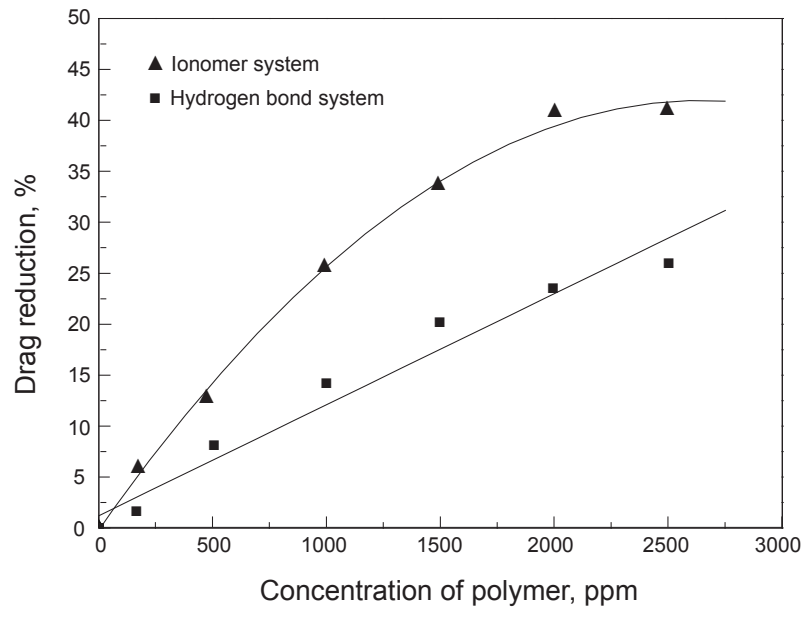

Fig. 10 The drag reduction vs. polymer concentration

its distribution of polymers are also an important factor. The accept polymer and donor polymer were dissolved in toluene with the same concentration of $0.01 \mathrm{~g} / \mathrm{mL}$. Using 515-type high-temperature gel permeation chromatography (Waters Corporation, USA), the molecular weight of polymer drag reducers was measured. The distribution of polymer of the ionomer system and the hydrogen bond system are shown in Fig.11.

Fig. 11 shows that the molecular weight distribution is a single symmetrical peak. The computer data analysis shows that the polymer has a high molecular weight. For hydrogen bonding complex: the number-average molecular weight (Mn) is 298,700 , the weight-average molecular weight $(\mathrm{Mw})$ is 382,600 and the dispersion degree is 2.63; For the blend complex: $\mathrm{Mn}$ is 3,593,587, Mw is 4,607,226 and the dispersion degree is 2.32 .

Using the testing and evaluation system, the relationship between the polymer molecular weight and drag reduction rate was obtained and is shown in Fig. 12.

As shown in Fig. 12, the drag reduction rate increased with increasing molecular weight. At low molecular weight, the drag reduction rate was quite different for different concentrations. This difference became smaller with

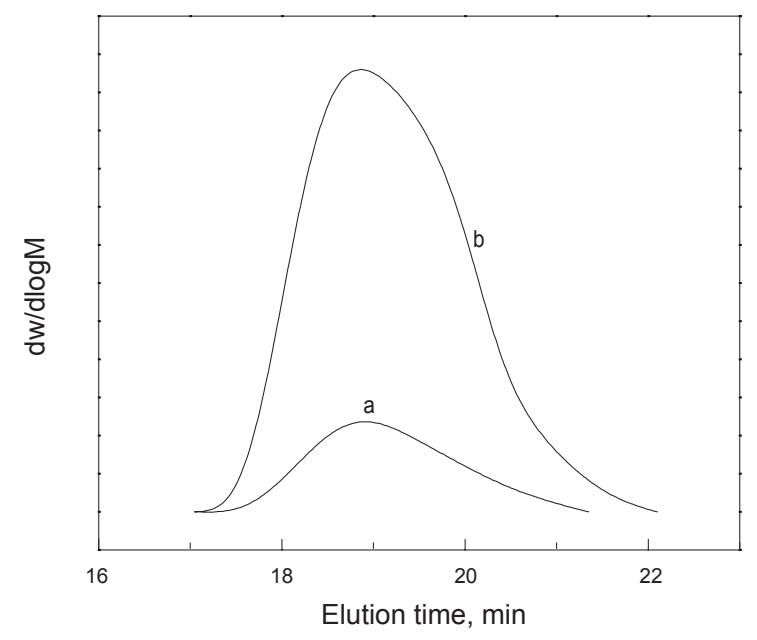

Fig. 11 The molecular weight distribution of polymer of ionomer system (a) and hydrogen bond system (b) 


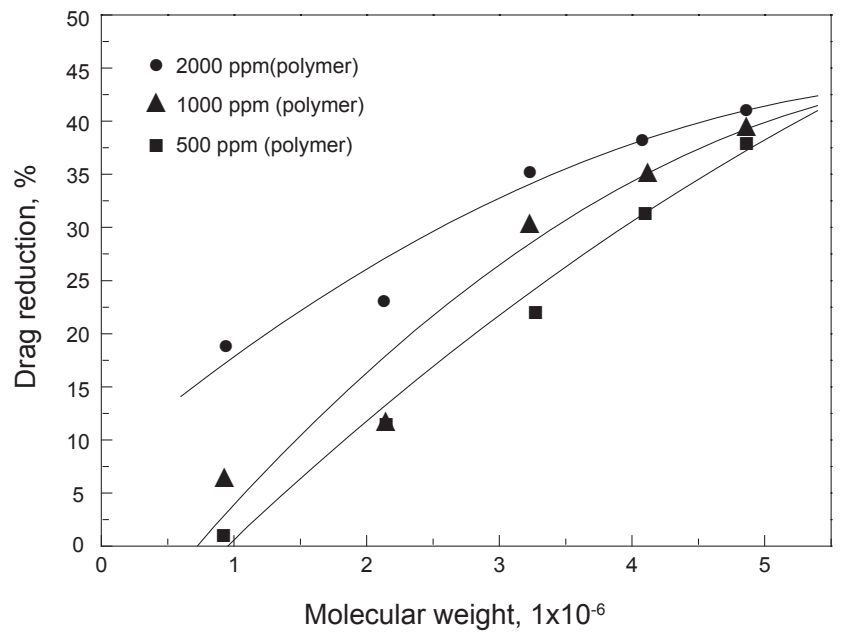

Fig. 12 The drag reduction rate vs. molecular weight of polymer

increasing molecular weight. When the molecular weight is about $5 \times 10^{6}$, the drag reduction rate of polymer with a concentration of $500 \mathrm{ppm}$ was similar to that of 2,000 ppm. These results indicated that increased molecular weight and concentrated molecular weight distribution of polymer were favorable to drag reduction.

\section{Shear resistance of ionomer system and hydrogen bond systems}

The shear resistance was tested using the tube pump transmission subsystem within the testing and evaluation system. New drag reduction tests were conducted in the diesel without addition of new DRA conditions relying on the gear pump subsystem cycle. So evaluation of the drag reduction and shear resistance of polymer system were achieved.

The experimental condition for shear resistance test was that the temperature was $25{ }^{\circ} \mathrm{C}$ and Reynolds number was 15,383 . Seven numbers were achieved under different concentrations of the three types of drag reduction agent. The relationship between drag reduction change and the shear number was shown in Fig. 13.

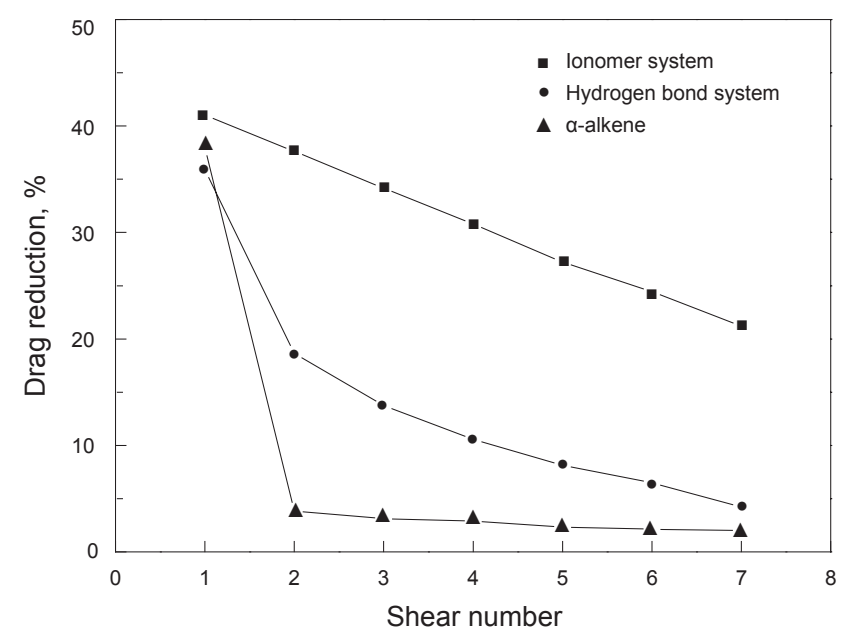

Fig. 13 Drag reduction rate with increase of shear number
From Fig. 13, we can see the drag reduction rate of polymer $\alpha$-alkene decreases from $38 \%$ to $2 \%$ after first shear, and no shear resistance was observed in the end. Although both the drag reduction rates of the ionomer and hydrogen bond systems decreases with increasing shear time, they exhibit excellent shear resistance. However, the hydrogen bond system shows a mutant site at the second shear. For ionomer system, the reduction rate decreases slowly, and the drag reduction rate is much larger than the two other systems. So, we deduce that the ionomer system has better potential industrial application than hydrogen bond system.

\section{Results}

1) When the ionomer system is formed by blending, it has strong ion-dipole interaction to form a "ladder" chain between the first order of pyridine functional groups and ions groups, leading to the shear resistance of the ionomer system. The shear resistance function was also observed in the hydrogen bond system. There are two parts for DRA, one part is first order pyridine of the chain compounds, and the other is long chain anionic compounds produced from the polymerization.

2) The synthesis of ionomer system is as follows: Taking ionization treatment on the bc-PDP of methyl methacrylate and lauryl methacrylate with methanol dissolved $\mathrm{NaOH}$, and then mixing with the tc-PDP of styrene, lauryl methacrylate and 2-vinyl pyridine at 1:1 (w/w). The hydrogen bond system was obtained by mixing the bc-PDP without the treatment with the tc-PDP at 1:1 (w/w).

3) Based on the relationship between Reynolds number and drag reduction performance, the ionomer polymer system shows advantages over the hydrogen bond system on drag reduction. The drag reduction efficiency of the ionomer is much better than that of the hydrogen bond system within the experimental concentrations.

4) Although the drag reduction rates of the two systems showed the same decreasing trend with increasing shear time, the hydrogen bond system decreases sharply at the second shear. But for the ionomer system, the reduction rate decreases slowly and smoothly. So the ionomer system may have better potential industrial application than hydrogen bond system owing to its better shear resistance and drag reduction.

\section{Acknowledgement}

This work is supported by the Basic Research Program of China (973 Program, Grant No. 2008CB617508).

\section{References}

Deng L and Liang D H. Online monitoring of the aggregation and fusion of DPPC/PA by static and dynamic light scattering. Acta Phys. Chim. Sin. 2010. 26(4): 862-868

Liu H, Song G J and Guan M. Characterization of $\alpha$-olefin polymer DRA via solution polymerization. Chinese Journal of Applied Chemistry. 2008. 25(8): 918-919 (in Chinese)

Liu P, Liu J L, Zhang D J and Zhang C Q. A comparative theoretical study of the reactivities of the $\mathrm{Al}^{+}$and $\mathrm{Cu}^{+}$ions toward methylamine and dimethylamine. International Journal of Quantum Chemistry. 2010. 110: 1583-1593 
Mei Y X. Development and construction of pipeline transportation in China. Communication and Transportation Systems Engineering and Information. 2005. 5(2): 108-111 (in Chinese)

Motier J F and Prilutski D J. Case histories of polymer drag reduction in crude oil lines. Pipe Line industry. 1985. (6): 33-37

Mowla D and Naderi A. Experimental study of drag reduction by a polymeric additive in slug two-phase flow of crude oil and air in horizontal pipe. Chemical Engineering Science. 2006. 61: 15491554

Pang Z J and Zhang C Q. Synthesis and properties of associating DRA by binary copolymerization base on lauryl methacrylate. Journal of Shandong University (Engineer Science). 2009. 39(5): 128-133 (in Chinese)

Peiffer D G. Drag reduction with novel hydrocarbon soluble polyampholytes. 1987. US Patent 4640954

Resende P R and Escudier M P. Numerical predictions and measurements of Reynolds normal stresses in turbulent pipe flow of polymers.
International Journal of Heat and Fluid Flow. 2006. ( 27): 204-219

Shui B Y, Liu B and Li G P. SY/T6578-2008 lab test method for the drag reduction performance of drag-reducing agent which applied in oil pipeline transportation(SY/T6578-2008). Beijing: Petroleum Industry Press (in Chinese)

Sun Y Z. The research and application of drag reduction technology in the pipeline. Petroleum Planning \& Engineering. 1996. (6): 27-28 (in Chinese)

Wilkens R J and Thomas D K. Multiphase drag reduction: Effect of eliminating slugs. Multiphase Flow. 2007. 33: 134-146

Zhao M Q, Cao J, Xi H M, et al. Influence of comminuting in liquid nitrogen on relative molecular mass of alkene polymer. Specialty Petrochemicals. 2007. 24( 5): 5-7 (in Chinese)

Zhu X Y, Zhang D J and Liu C B. Theoretical study of the structural characterizations of n-alkylpyridinium cations and their ion-pairs with some anions. Acta Chimica Sinica. 2007-23-009 (in Chinese)

(Edited by Zhu Xiuqin) 